\title{
Scattering Of SH Waves By a Circular Inclusion Under a Variable Circular-Arc Hill
}

\author{
Gao Ling ${ }^{1, ~ a, ~ L i u ~ Q i f a n g ~}{ }^{1, b}$ \\ ${ }^{1}$ Institute of Engineering Mechanics, China Earthquake Administration; Key Laboratory of \\ Earthquake Engineering and Engineering Vibration, China Earthquake Administration, China \\ a472847425@qq.com, b357237916@qq.com
}

\begin{abstract}
Keywords: Wave functions expansion. Underground inclusion. Circular-arc hill. Surface ground motion
\end{abstract}

Abstract. An inclusion under a hill would amplify the ground motion tremendously. However, Few analytic solutions achieved for this problem have limitations on a special semi-cylindrical hill or a underground cavity. Here we derive one for a new model with the wave functions expansion and auxiliary functions technique. It is reduced to solving a set of infinite linear algebraic equation using Fourier expansion for auxiliary functions based on boundary condition. The solution can be degenerated to the ones of the model ignoring the hill or the inclusion. Finally, numerical solutions are obtained by truncation of the infinite equations. The results indicate that the ground motion could be seen as the superposition of the effect of the inclusion and the hill qualitatively; and when the inclusion degenerates to a cavity, amplification frequencies on the hilly boundary can be obtained by the wave speed in half-space and the vertical distance between the flat surface and the tunnel. The influence of softness and hardness of inclusion and incident angles is also discussed here.

\section{Introduction}

Analyses show that special surface topographies (e.g. hills, alluvial valleys, canyons) and underground topographies (e.g. underground inclusions and cavities) have a complex effect on ground motion. This paper will put both types of topographies together, which investigated here would be the case of a variable circular-arc hill on top of an underground circular inclusion.

To study the problem, few previous analytical solutions related to diffraction of SH waves by a hill and a subsurface inclusion in the elastic half-space will first be summarized here. The diffraction of SH waves by a variable circular-arc hill with a inside concentric circular cavity has been solved [1]. The diffraction of SH waves by a semi-cylindrical hill above a subsurface cavity has also been studied [2].

All of the investigations above were confined to a special semi-cylindrical hill or a subsurface circular cavity concentric with the hill, and they analyzed the effect without comparing with the degraded situations ignoring the underground and surface topography respectively. In this paper we obtain the analytical solutions of the scattering of $\mathrm{SH}$ wave by a variable circular-arc hill above a subsurface inclusion with wave functions expansion, and it can be degraded to the ones without the inclusion or without the hill.

\section{Mathematical model}

The model as shown in fig 1 consists of an elastic, isotropic and homogeneous half-space and a circular-arc hill of radius al above an inclusion of radius $a_{2}$. The free surface of the half-space consists of a flat surface $\Gamma$ and a circular-arc hilly boundary $L$. The boundary of the inclusion is marked as $C$. The vertical distance between origin $o_{1}$ and the flat surface is $d_{1}$; the distance between origin $o_{1}$ and origin $o_{2}$ is $d_{2}$; and $d_{3}=d_{1}+d_{2}$ presents the buried depth of origin $o_{2}$. The height and half-width of the hill is $h$ and $b$. The ratio of $h / b$ is named as height-to-width ratio. The material properties are given by the shear modulus $\mu$ and the velocity $c$. The subscript 1,2 on $\mu, c$ designate these constants in the half-space and that in the inclusion. 
The incident SH wave $u^{(i)}$ with incident angle $\alpha$ can be expressed in the Cartesian coordinate $x$ - $o-y$ as :

$$
u^{(i)}(x, y)=u_{0} e^{-i \omega\left(-\frac{x}{c_{x}}-\frac{y}{c_{y}}\right)}
$$

Where time factor $\exp (-\mathrm{i} \omega t)$ was omitted, and it is same for the following equations; $c_{x}=c / \sin \alpha$ and $c_{y}=c / \cos \alpha$ are the phase velocities in the $x$ and $y$ direction, respectively; and $\omega$ and $u_{0}$ are the circular frequency and amplitude of the incident $\mathrm{SH}$ wave, respectively.

The total displacement $u$ in the half-space must satisfy the wave equation

$$
\frac{\partial u^{2}}{\partial r^{2}}+\frac{\partial u}{r \partial r}+\frac{\partial^{2} u}{r^{2} \partial \theta^{2}}+\frac{\omega^{2}}{c^{2}} u=0
$$

The traction-free boundary conditions are

$$
\begin{aligned}
& \sigma_{r z}=0,(r, \theta) \in \Gamma \\
& \sigma_{\theta z}=0,(r, \theta) \in L
\end{aligned}
$$

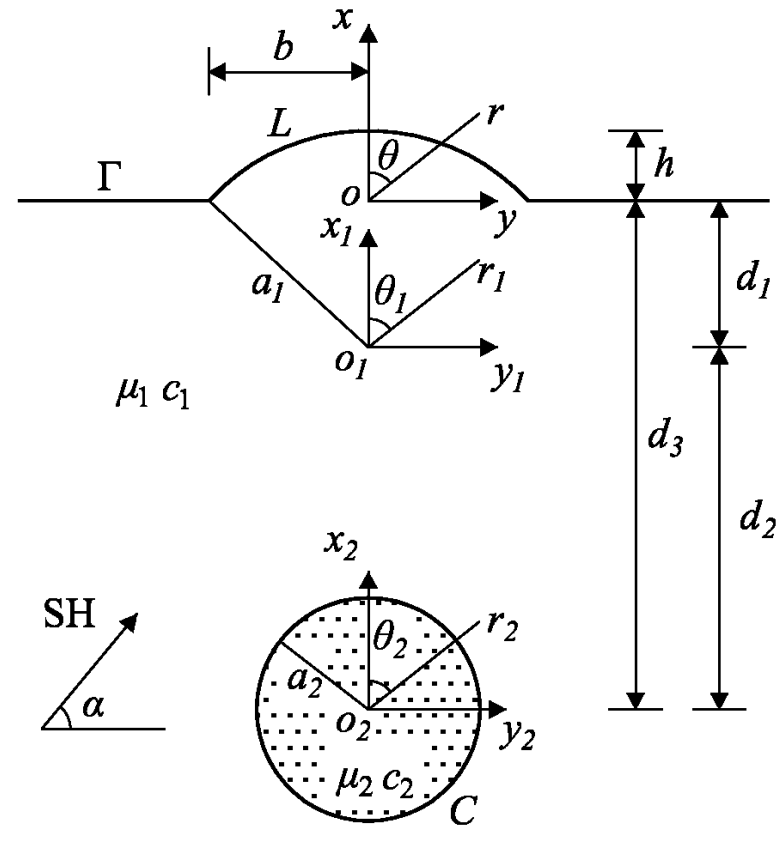

Fig.1 Model given by

$$
\sigma_{r z}=\mu \frac{\partial u}{\partial r}, \sigma_{\theta z}=\frac{\mu}{r} \frac{\partial u}{\partial \theta}
$$

The model is divided into three parts as shown in fig 2, one is circular region II including the hill, whose upper and lower boundaries are $L$ and $\bar{L}$, one is circular region III including the inclusion, whose boundary is $C$, and the rest of the model is region I, which has common boundaries $\bar{L}$ and $C$ with region II and III.
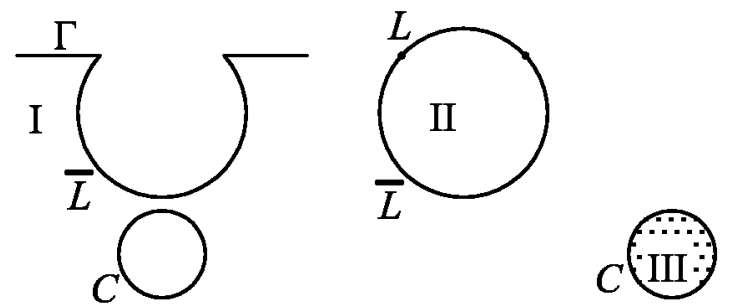

Fig. 2 The division of the solution domain

The displacement field in the three regions can be expressed by

$$
u= \begin{cases}u^{(f)}+u^{(1)}+u^{(2)} & (r, \theta) \in \mathrm{I} \\ u^{(3)} & (r, \theta) \in \mathrm{II} \\ u^{(4)} & (r, \theta) \in \mathrm{III}\end{cases}
$$

Where $u^{(f)}$ represents the free field displacement in the half-space, $u^{(1)}$ represents the scattering displacement from boundary $\bar{L}, u^{(2)}$ represents the scattering displacement from boundary $C, u^{(3)}$ represents the scattering displacement in the region II, and $u^{(4)}$ represents the scattering displacement in the region III.

For the convenience of the problem, we create three cylindrical coordinates system as shown in fig 1: $(r, \theta),\left(r_{1}, \theta_{1}\right),\left(r_{2}, \theta_{2}\right)$. Finally, the displacement and stress continuity conditions on the interface $\bar{L}$ and $C$ need satisfy the following equations, respectively.

$$
u^{(\mathrm{II})}\left(r_{1}, \theta_{1}\right)=u^{(\mathrm{II})}\left(r_{1}, \theta_{1}\right),\left(r_{1}, \theta_{1}\right) \in \bar{L}
$$




$$
\begin{gathered}
\sigma_{r_{1} z}^{(\mathrm{I})}\left(r_{1}, \theta_{1}\right)=\sigma_{r_{1} z}^{(\mathrm{II})}\left(r_{1}, \theta_{1}\right),\left(r_{1}, \theta_{1}\right) \in \bar{L} \\
u^{(\mathrm{I})}\left(r_{2}, \theta_{2}\right)=u^{(\mathrm{IIII})}\left(r_{2}, \theta_{2}\right),\left(r_{2}, \theta_{2}\right) \in C \\
\sigma_{r_{2} z}^{(\mathrm{I})}\left(r_{2}, \theta_{2}\right)=\sigma_{r_{2} z}^{(\mathrm{III})}\left(r_{2}, \theta_{2}\right),\left(r_{2}, \theta_{2}\right) \in C
\end{gathered}
$$

Where superscript I, II, III represent the corresponding region.

\section{Analytical solutions}

In this section the displacement and stress expression in region I, II, III in the coordinate system $\left(r_{1}, \theta_{1}\right),\left(r_{2}, \theta_{2}\right)$ will be obtained for the convenience of solving the boundary equations.

Region I. The displacement $u^{(f)}$ can be expressed by

$$
u^{(f)}=u^{(i)}+u^{(r)}
$$

Where $u^{(i)}$ represents the incident SH wave, $u^{(r)}$ represents the reflected SH wave on the flat ground surface, $u^{(r)}$ can be written as

$$
u^{(r)}(x, y)=u_{0} e^{-i \omega\left(\frac{x}{c_{x}}-\frac{y}{c_{y}}\right)}
$$

Inserting $u^{(i)}, u^{(r)}$ into equation (10) leads to

$$
u^{(f)}\left(r_{1}, \theta_{1}\right)=u_{0} \xi_{1}^{-1} \mathrm{e}^{\mathrm{i} \beta_{1} r_{1} \cos \theta_{4}}+u_{0} \xi_{1} \mathrm{e}^{\mathrm{i} \beta_{1} r_{1} \cos \theta_{5}}
$$

Where $\beta_{1}=\omega / c_{1}, \theta_{4}=\pi / 2-\alpha-\theta_{1}, \theta_{5}=\pi / 2+\alpha-\theta_{1}, \xi_{1}=\mathrm{e}^{\mathrm{i} \beta_{1} d_{1} \sin \alpha}$.

By transform formula

$$
\mathrm{e}^{\mathrm{i} \beta r \cos \theta}=\sum_{n=0}^{\infty} \varepsilon_{n} \mathrm{i}^{n} J_{n}\left(\beta_{1} r\right) \cos n \theta
$$

Eq. (12) can be written as

$$
u^{(f)}\left(r_{1}, \theta_{1}\right)=u_{0} \sum_{n=0}^{\infty} \varepsilon_{n} \mathrm{i}^{n} \kappa_{1 n} J_{n}\left(\beta_{1} r_{1}\right)
$$

Where $\varepsilon_{0}=1, \varepsilon_{1,2,3 \ldots n}=2$, and $J_{n}(x)$ are the Bessel functions of the first kind with argument $x$ and order $n$, and

$$
\kappa_{i n}=\left[\xi_{i}^{-1} \cos n\left(\frac{\pi}{2}-\alpha\right)+\xi_{i} \cos n\left(\frac{\pi}{2}+\alpha\right)\right] \cos n \theta_{i}+\left[\xi_{i}^{-1} \sin n\left(\frac{\pi}{2}-\alpha\right)+\xi_{i} \sin n\left(\frac{\pi}{2}+\alpha\right)\right] \sin n \theta_{i}
$$

Inserting Eq. (14) into Eq. (5) leads to

$$
\sigma_{r_{1} z}^{(f)}\left(r_{1}, \theta_{1}\right)=\frac{\sigma_{0}}{\beta_{1} r_{1}} \sum_{n=0}^{\infty} \varepsilon_{n} i^{n} \kappa_{1 n} R_{n}\left(\beta_{1} r_{1}\right)
$$

Where $\sigma_{0}=\mu_{1} \beta_{1} u_{0}$, representing the stress amplitude of the incident wave, and

$$
R_{n}\left(\beta_{1} r_{1}\right)=n J_{n}\left(\beta_{1} r_{1}\right)-\beta_{1} r_{1} J_{n+1}\left(\beta_{1} r_{1}\right) .
$$

Similarly, $u^{(f)}, \sigma_{r_{2} z}^{(f)}$ in the coordinate system $\left(r_{2}, \theta_{2}\right)$ can be expressed as

$$
\begin{gathered}
u^{(f)}\left(r_{2}, \theta_{2}\right)=u_{0} \sum_{n=0}^{\infty} \varepsilon_{n} \dot{i}^{n} \kappa_{2 n} J_{n}\left(\beta_{1} r_{2}\right) \\
\sigma_{r_{2} z}^{(f)}\left(r_{2}, \theta_{2}\right)=\frac{\sigma_{0}}{\beta_{1} r_{2}} \sum_{n=0}^{\infty} \varepsilon_{n} \mathrm{i}^{n} \kappa_{2 n} R_{n}\left(\beta_{1} r_{2}\right) \kappa_{2 n}
\end{gathered}
$$

Where $\xi_{2}=\mathrm{e}^{\mathrm{i} \beta_{1} d_{3} \sin \alpha}$

Eq. (14) (17) strictly satisfy The traction-free boundary condition (4).

Next,$u^{(1)}$ in coordinate system $(r, \theta)$ is obtained with satisfying equation (2) and the boundary condition (4):

$$
u^{(1)}(r, \theta)=u_{0} \sum_{n=0}^{\infty} H_{n}^{(1)}(\beta r)\left(A_{n} \delta_{n}^{(1)} \cos n \theta+B_{n} \delta_{n}^{(2)} \sin n \theta\right)
$$

Where $A_{n}$ and $B_{n}$ are constants to be determined, $\delta_{n}(1)=1+(-1) n, \delta_{n}(1)=1-(-1) n$, and $\mathrm{H}_{n}^{(1)}(x)$ are the Hankel functions of the first kind order with argument $x$ and order $n$. 
This scattered field has also automatically satisfied the traction-free boundary condition (4).

To transform the Hankel functions in coordinate system $(r, \theta)$ into those in coordinate system $\left(r_{1}, \theta_{1}\right)$, the following exterior region form of Graf's addition theorem is used [3]:

$$
H_{n}^{(1)}\left(\beta_{1} r\right)\left\{\begin{array}{c}
\cos n \theta \\
\sin n \theta
\end{array}\right\}=(-1)^{n} \sum_{m=0}^{\infty} \frac{\varepsilon_{m}}{2} H_{m}^{(1)}\left(\beta_{1} r_{1}\right) \times\left\{\begin{array}{l}
{\left[J_{m+n}\left(\beta_{1} d_{1}\right)+(-1)^{n} J_{m-n}\left(\beta_{1} d_{1}\right)\right] \cos m \theta_{1}} \\
-\left[J_{m+n}\left(\beta_{1} d_{1}\right)-(-1)^{n} J_{m-n}\left(\beta_{1} d_{1}\right)\right] \sin m \theta_{1}
\end{array}\right\}
$$

Inserting equation (19) into equation (18) leads to

$$
u^{(1)}\left(r_{1}, \theta_{1}\right)=u_{0} \sum_{m=0}^{\infty} H_{m}^{(1)}\left(\beta_{1} r_{1}\right)\left(A_{m}^{\prime} \cos m \theta_{1}-B_{m}^{\prime} \sin m \theta_{1}\right)
$$

Where

$$
\begin{aligned}
& A_{m}^{\prime}=\frac{\varepsilon_{m}}{2} \sum_{n=0}^{\infty}(-1)^{n} \delta_{n}^{(1)}\left[J_{m+n}\left(\beta_{1} d_{1}\right)+(-1)^{n} J_{m-n}\left(\beta_{1} d_{1}\right)\right] A_{n} \\
& B_{m}^{\prime}=\frac{\varepsilon_{m}}{2} \sum_{n=0}^{\infty}(-1)^{n} \delta_{n}^{(2)}\left[J_{m+n}\left(\beta_{1} d_{1}\right)-(-1)^{n} J_{m-n}\left(\beta_{1} d_{1}\right)\right] B_{n}
\end{aligned}
$$

Inserting equation (20) into equation (5) leads to

$$
\sigma_{r_{1} z}^{(1)}\left(r_{1}, \theta_{1}\right)=\frac{\sigma_{0}}{r_{1} \beta_{1}} \sum_{m=0}^{\infty} T_{m}\left(\beta_{1} r_{1}\right)\left(A_{m}^{\prime} \cos m \theta_{1}-B_{m}^{\prime} \sin m \theta_{1}\right)
$$

Where $T_{m}\left(\beta_{1} r\right)=\beta_{1} H_{m}^{(1)}\left(\beta_{1} r_{1}\right)-\beta_{1} r_{1} H_{m+1}^{(1)}\left(\beta_{1} r_{1}\right)$.

Similarly, $u^{(1)}$ in coordinate system $\left(r_{2}, \theta_{2}\right)$ can be obtained by the interior form of the Graf's addition theorem:

$$
u^{(1)}\left(r_{2}, \theta_{2}\right)=u_{0} \sum_{m=0}^{\infty} J_{m}\left(r_{2}, \theta_{2}\right)\left(A_{m}^{\prime \prime} \cos m \theta_{2}-B_{m}^{\prime \prime} \sin m \theta_{2}\right)
$$

Where

$$
\begin{aligned}
& A_{m}^{\prime \prime}=\frac{\varepsilon_{m}}{2} \sum_{n=0}^{\infty}(-1)^{n} \delta_{n}^{(1)}\left[H_{m+n}^{(1)}\left(\beta_{1} d_{3}\right)+(-1)^{n} H_{m-n}^{(1)}\left(\beta_{1} d_{3}\right)\right] A_{n} \\
& B_{m}^{\prime \prime}=\frac{\varepsilon_{m}}{2} \sum_{n=0}^{\infty}(-1)^{n} \delta_{n}^{(2)}\left[H_{m+n}^{(1)}\left(\beta_{1} d_{3}\right)-(-1)^{n} H_{m-n}^{(1)}\left(\beta_{1} d_{3}\right)\right] B_{n}
\end{aligned}
$$

From $u^{(1)}\left(r_{2}, \theta_{2}\right), \sigma_{r_{2} z}^{(1)}\left(r_{2}, \theta_{2}\right)$ can be obtained:

$$
\sigma_{r_{2} z}^{(1)}\left(r_{2}, \theta_{2}\right)=\frac{\sigma_{0}}{r_{2} \beta_{1}} \sum_{m=0}^{\infty} R_{m}\left(\beta_{1} r_{2}\right)\left(A_{m}^{\prime \prime} \cos m \theta_{2}-B_{m}^{\prime \prime} \sin m \theta_{2}\right)
$$

To obtain the scattered wave by the inclusion, we use the image method. The model is built as fig 3 , the origin $o_{3}$ is the virtual source of the origin $o_{2}$, and corresponding coordinate systems are $\left(x_{3}, y_{3}\right)$ and $\left(r_{3}, \theta_{3}\right)$.

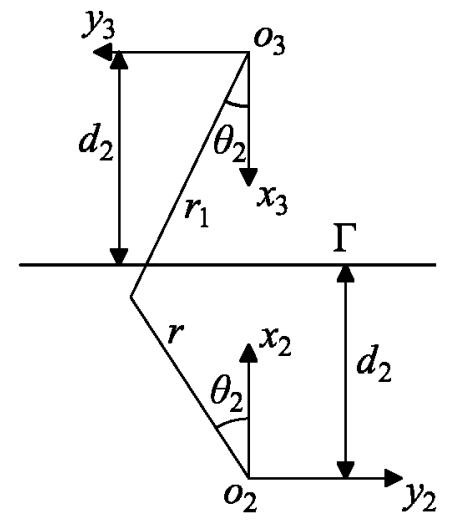

Fig. 3 diagram of scattered wave of virtual source

In the approach, $u^{(2)}$ is expressed as

$$
u^{(2)}=u^{(a)}+u^{(b)}
$$


Where $u^{(a)}$ represents the scattered displacement by source $o_{2}, u^{(b)}$ represents the scattered displacement by source $o_{3}$.

The general solution of equation (2) is

$$
u^{(a)}\left(r_{2}, \theta_{2}\right)=u_{0} \sum_{n=0}^{\infty} H_{n}^{(1)}\left(\beta_{1} r_{2}\right)\left(C_{n} \cos n \theta_{2}+D_{n} \sin n \theta_{2}\right)
$$

Where $C_{n}, D_{n}$ are the constants to be determined.

Similarly, $u^{(b)}$ satisfying the wave function (2) can also be obtained as

$$
u^{(b)}\left(r_{3}, \theta_{3}\right)=u_{0} \sum_{n=0}^{\infty} H_{n}^{(1)}\left(\beta_{1} r_{3}\right)\left(C_{n} \cos n \theta_{3}-D_{n} \sin n \theta_{3}\right)
$$

$u^{(2)}$ satisfies the traction-free boundary condition automatically(Yuan 1999).

From the interior Graf's addition theorem, $u^{(2)}$ in coordinate system $\left(r_{1}, \theta_{1}\right)$ can be obtained by transforming coordinate system $\left(r_{2}, \theta_{2}\right)$ and $\left(r_{3}, \theta_{3}\right)$ into $\left(r_{1}, \theta_{1}\right)$, respectively.

$$
u^{(2)}\left(r_{1}, \theta_{1}\right)=\sum_{m=0}^{\infty} J_{m}\left(\beta_{1} r_{1}\right)\left(C_{m}^{\prime \prime} \cos m \theta_{1}+D_{m}^{\prime \prime} \sin m \theta_{1}\right)
$$

Where $d_{4}=d_{1}+d_{3}$

$$
\begin{aligned}
& C_{m}^{\prime \prime}=\frac{\varepsilon_{m}}{2} \sum_{n=0}^{\infty}\left\{(-1)^{m}\left[(-1)^{n} H_{m-n}^{(1)}\left(\beta_{1} d_{2}\right)+H_{m+n}^{(1)}\left(\beta_{1} d_{2}\right)\right]+\left[(-1)^{n} H_{m-n}^{(1)}\left(\beta_{1} d_{4}\right)+H_{m+n}^{(1)}\left(\beta_{1} d_{4}\right)\right]\right\} C_{n} \\
& D_{m}^{\prime \prime}=\frac{\varepsilon_{m}}{2} \sum_{n=0}^{\infty}\left\{(-1)^{m}\left[(-1)^{n} H_{m-n}^{(1)}\left(\beta_{1} d_{2}\right)-H_{m+n}^{(1)}\left(\beta_{1} d_{2}\right)\right]-\left[(-1)^{n} H_{m-n}^{(1)}\left(\beta_{1} d_{4}\right)-H_{m+n}^{(1)}\left(\beta_{1} d_{4}\right)\right]\right\} D_{n}
\end{aligned}
$$

Inserting equation (28) into (5) leads to

$$
\begin{aligned}
\sigma_{r_{1} z}^{(2)}\left(r_{1}, \theta_{1}\right)= & \frac{\sigma_{0}}{\beta_{1} r_{1}} \sum_{m=0}^{\infty} R_{m}\left(\beta_{1} r_{1}\right) \\
& \times\left(C_{m}^{\prime \prime} \cos m \theta_{1}+D_{m}^{\prime \prime} \sin m \theta_{1}\right)
\end{aligned}
$$

To obtain $u^{(2)}, \sigma_{r_{2} z}^{(2)}$ in coordinate system $\left(r_{2}, \theta_{2}\right)$, from the interior Graf's addition theorem, we obtain $u^{(2)}\left(r_{2}, \theta_{2}\right)=u_{0} \sum_{m=0}^{\infty} H_{m}^{(1)}\left(\beta_{1} r_{2}\right)\left(C_{m} \cos m \theta_{2}+D_{m} \sin m \theta_{2}\right)+u_{0} \sum_{m=0}^{\infty} J_{m}\left(\beta_{1} r_{2}\right)\left(C_{m}^{\prime} \cos m \theta_{2}+D_{m}^{\prime} \sin m \theta_{2}\right)$

where

$$
\begin{aligned}
& C_{m}^{\prime}=\frac{\varepsilon_{m}}{2} \sum_{n=0}^{\infty}\left[H_{m+n}^{(1)}\left(2 \beta_{1} d_{2}\right)+(-1)^{n} H_{m-n}^{(1)}\left(2 \beta_{1} d_{2}\right)\right] C_{n} \\
& D_{m}^{\prime}=\frac{\varepsilon_{m}}{2} \sum_{n=0}^{\infty}\left[H_{m+n}^{(1)}\left(2 \beta_{1} d_{3}\right)-(-1)^{n} H_{m-n}^{(1)}\left(2 \beta_{1} d_{3}\right)\right] D_{n}
\end{aligned}
$$

Inserting equation (29) into (5) leads to

$$
\begin{aligned}
\sigma_{r_{2} z}^{(2)}\left(r_{2}, \theta_{2}\right) & =\frac{\sigma_{0}}{\beta_{1} r_{2}} \sum_{m=0}^{\infty} T_{m}\left(\beta_{1} r_{2}\right)\left(C_{m} \cos m \theta_{2}+D_{m} \sin m \theta_{2}\right) \\
& +\frac{\sigma_{0}}{\beta_{1} r_{2}} \sum_{m=0}^{\infty} R_{m}\left(\beta_{1} r_{2}\right)\left(C_{m}^{\prime} \cos m \theta_{2}+D_{m}^{\prime} \sin m \theta_{2}\right)
\end{aligned}
$$

Region II. The general solution of Eq. (2) in region II can be expressed as

$$
u^{(3)}\left(r_{1}, \theta_{1}\right)=u_{0} \sum_{m=0}^{\infty} J_{m}\left(\beta_{1} r_{1}\right)\left(E_{m} \cos m \theta_{1}+F_{m} \sin m \theta_{1}\right)
$$

where $E_{m}, F_{m}$ are constants to be determined.

$\sigma_{r_{1} z}^{(3)}\left(r_{1}, \theta_{1}\right)$ can be obtained as follows

$$
\sigma_{r_{1} z}^{(3)}\left(r_{1}, \theta_{1}\right)=\frac{\sigma_{0}}{\beta_{1} r_{1}} \sum_{m=0}^{\infty} R_{m}\left(\beta_{1} r_{1}\right)\left(E_{m} \cos m \theta_{1}+F_{m} \sin m \theta_{1}\right)
$$


Region III. The general solution of equation (2) in region III can expressed as

$$
u^{(4)}\left(r_{2}, \theta_{2}\right)=u_{0} \sum_{m=0}^{\infty} J_{m}\left(\beta_{2} r_{2}\right)\left(G_{m} \cos m \theta_{2}+H_{m} \sin m \theta_{2}\right)
$$

Where $G_{m}, H_{m}$ are constants to be determined.

$\sigma_{r_{2} z}^{(4)}\left(r_{2}, \theta_{2}\right)$ can be obtained as follows

$$
\sigma_{r_{2} z}^{(4)}\left(r_{2}, \theta_{2}\right)=\frac{\sigma_{0}}{\beta_{2} r_{2}} \sum_{m=0}^{\infty} R_{m}\left(\beta_{2} r_{2}\right)\left(G_{m} \cos m \theta_{2}+H_{m} \sin m \theta_{2}\right)
$$

\section{Solving equations}

From boundary conditions, the boundary $L$ meets the stress-free condition, and the boundary $\bar{L}$ meets the continuity condition. To solve the mixed boundary value problem, we define the functions $\varphi\left(\theta_{1}\right)$ and $\psi\left(\theta_{1}\right)$ as follows :

$$
\begin{gathered}
\varphi\left(\theta_{1}\right)=\left\{\begin{array}{l}
\sigma_{r z}^{(3)}\left(a_{1}, \theta_{1}\right),-\varphi+2 k \pi \leq \theta_{1} \leq \varphi+2 k \pi \\
\sigma_{r_{1} z}^{(3)}\left(a_{1}, \theta_{1}\right)-\sigma_{r_{1} z}^{(f)}\left(a_{1}, \theta_{1}\right) \\
-\sigma_{r_{1} z}^{(1)}\left(a_{1}, \theta_{1}\right)-\sigma_{r_{1} z}^{(2)}\left(a_{1}, \theta_{1}\right), \quad \text { other } \\
k=0, \pm 1, \pm 2 \ldots
\end{array}\right. \\
\psi\left(\theta_{1}\right)=\left\{\begin{array}{l}
0, \quad-\varphi+2 k \pi \leq \theta_{1} \leq \varphi+2 k \pi \\
u^{(3)}\left(a_{1}, \theta_{1}\right)-u^{(f)}\left(a_{1}, \theta_{1}\right) \\
-u^{(1)}\left(a_{1}, \theta_{1}\right)-u^{(2)}\left(a_{1}, \theta_{1}\right), \quad \text { other } \\
k=0, \pm 1, \pm 2 \ldots
\end{array}\right.
\end{gathered}
$$

From the boundary condition of region II, we can obtain the condition as follows:

$$
\begin{gathered}
\varphi\left(\theta_{1}\right)=0,-\pi+2 k \pi \leq \theta_{1} \leq \pi+2 k \pi \\
\psi\left(\theta_{1}\right)=0,-\pi+2 k \pi \leq \theta_{1} \leq \pi+2 k \pi
\end{gathered}
$$

Make their Fourier series expansions over $[-\pi, \pi]$, and let the coefficients of the series equal zero. Rearrange the equation yields

$$
\begin{gathered}
R_{n}\left(\beta_{1} a_{1}\right) E_{n}-\sum_{n=0}^{\infty}\left[A_{m}^{\prime} T_{m}\left(\beta_{1} a_{1}\right)+C_{m}^{\prime \prime} R_{m}\left(\beta_{1} a_{1}\right)\right] \lambda_{m n}=\sum_{m=0}^{\infty} R_{m}\left(\beta_{1} a_{1}\right) U_{m} \lambda_{m n} \\
\sum_{k=0}^{\infty} J_{k}\left(\beta_{1} a_{1}\right) E_{k} \lambda_{k n}-\sum_{m=0}^{\infty}\left[H_{m}^{(1)}\left(\beta_{1} a_{1}\right) A_{m}^{\prime}+J_{m}\left(\beta_{1} a_{1}\right) C_{m}^{\prime \prime}\right] \lambda_{m n}=\sum_{m=0}^{\infty} J_{m}\left(\beta_{1} a_{1}\right) U_{m} \lambda_{m n} \\
n=0,1,2,3 \ldots \\
\sum_{m=0}^{\infty}\left[B_{m}^{\prime} T_{m}\left(\beta_{1} a_{1}\right)-D_{m}^{\prime \prime} R_{m}\left(\beta_{1} a_{1}\right)\right] \mu_{m n}+R_{n}\left(\beta_{1} \alpha_{1}\right) F_{n}=\sum_{m=0}^{\infty} R_{m}\left(\beta_{1} a_{1}\right) V_{m} \mu_{m n} \\
\sum_{m=0}^{\infty}\left[H_{m}^{(1)}\left(\beta_{1} a_{1}\right) B_{m}^{\prime}-J_{m}\left(\beta_{1} a_{1}\right) D_{m}^{\prime \prime}\right] \mu_{m n}+\sum_{k=0}^{\infty} J_{k}\left(\beta_{1} a_{1}\right) F_{k} \mu_{k n}=\sum_{m=0}^{\infty} J_{m}\left(\beta_{1} a_{1}\right) V_{m} \mu_{m} \\
n=1,2,3 \ldots
\end{gathered}
$$

Where

$$
\lambda_{m n}=\left\{\begin{array}{lr}
\frac{\pi-\varphi}{\pi}, & n=m=0 \\
\frac{1}{\pi}\left(\frac{-\sin 2 n \varphi}{2 n}+\pi-\varphi\right), & n=m \neq 0 \\
\frac{\varepsilon_{n}}{2 \pi}\left(\frac{-\sin (m+n) \varphi}{m+n}-\frac{\sin (m-n) \varphi}{m-n}\right), & n \neq m
\end{array}\right.
$$




$$
\mu_{m n}=\left\{\begin{array}{lr}
0, & n=m=0 \\
\frac{1}{\pi}\left(\frac{\sin 2 n \varphi}{2 n}+\pi-\varphi\right), & n=m \neq 0 \\
\frac{\varepsilon_{n}}{2 \pi}\left(\frac{\sin (m+n) \varphi}{m+n}-\frac{\sin (m-n) \varphi}{m-n}\right), & n \neq m
\end{array}\right.
$$

From the continuity condition on the boundary $C$, inserting equations (16) and (17) (23) (30) (34) into equation (8) and (9) respectively, the equations to determine the unknown constants can be obtained as follows:

$$
\begin{aligned}
& \gamma R_{n}\left(\beta_{2} a_{2}\right) G_{n}-R_{n}\left(\beta_{1} a_{2}\right) A_{n}^{\prime \prime}-T_{n}\left(\beta_{1} a_{2}\right) C_{n}-R_{n}\left(\beta_{1} a_{2}\right) C_{n}^{\prime}=R_{n}\left(\beta_{1} a_{2}\right) W_{n} \\
& J_{n}\left(\beta_{2} a_{2}\right) G_{n}-J_{n}\left(\beta_{1} a_{2}\right) A_{n}^{\prime \prime}-H_{n}^{(1)}\left(\beta_{1} a_{2}\right) C_{n}-J_{n}\left(\beta_{1} a_{2}\right) C_{n}^{\prime}=J_{n}\left(\beta_{1} a_{2}\right) W_{n} \\
& \gamma R_{n}\left(\beta_{2} a_{2}\right) H_{n}+R_{n}\left(\beta_{1} a_{2}\right) B_{n}^{\prime \prime}-T_{n}\left(\beta_{1} a_{2}\right) D_{n}-R_{n}\left(\beta_{1} a_{2}\right) D_{n}^{\prime}=R_{n}\left(\beta_{1} a_{2}\right) X_{n} \\
& J_{n}\left(\beta_{2} a_{2}\right) H_{n}+J_{n}\left(\beta_{1} a_{2}\right) B_{n}^{\prime \prime}-H_{n}^{(1)}\left(\beta_{1} a_{2}\right) D_{n}-J_{n}\left(\beta_{1} a_{2}\right) D_{n}^{\prime}=J_{n}\left(\beta_{1} a_{2}\right) X_{n}
\end{aligned}
$$

Rearranging equations (37) (44) leads to a set of infinite linear algebraic equations as follows:

$$
\begin{gathered}
\sum_{n=0}^{\infty} \Delta_{m n} A_{n}+\sum_{n=0}^{\infty} \Delta_{m n}^{\prime} C_{n}=\sum_{n=0}^{\infty} U_{n}\left[J_{n}\left(\beta_{1} a_{1}\right) \lambda_{n m}-R_{n}\left(\beta_{1} a_{1}\right) P_{n m}\right] \\
\sum_{n=0}^{\infty} \Delta_{m n}^{\prime \prime} A_{n}+\Phi_{m} C_{m}+\sum_{n=0}^{\infty} \Delta_{m n}^{\prime \prime \prime} C_{n}=\left[\gamma R_{m}\left(\beta_{2} a_{2}\right) J_{m}\left(\beta_{1} a_{2}\right)-J_{m}\left(\beta_{2} a_{2}\right) R_{m}\left(\beta_{1} a_{2}\right)\right] W_{m} \\
m=0,1,2,3 \ldots \\
\sum_{n=0}^{\infty} \Lambda_{m n} B_{n}+\sum_{n=0}^{\infty} \Lambda_{m n}^{\prime} D_{n}=\sum_{n=0}^{\infty} V_{n}\left[J_{n}\left(\beta_{1} a_{1}\right) \mu_{n m}-R_{n}\left(\beta_{1} a_{1}\right) Q_{n m}\right] \\
\sum_{n=0}^{\infty} \Lambda_{m n}^{\prime \prime} B_{n}+\Phi_{m}^{\prime} D_{m}+\sum_{n=0}^{\infty} \Lambda_{m n}^{\prime \prime \prime} D_{n}=\left[J_{m}\left(\beta_{2} a_{2}\right) R_{m}\left(\beta_{1} a_{2}\right)-\gamma J_{m}\left(\beta_{1} a_{2}\right) R_{m}\left(\beta_{2} a_{2}\right)\right] X_{m} \\
m=1,2,3 \ldots
\end{gathered}
$$

Where

$$
\begin{gathered}
\Delta_{m n}=\frac{\delta_{n}^{(1)}}{2} \sum_{k=0}^{\infty} \varepsilon_{k}\left[(-1)^{n} J_{k+n}\left(\beta_{1} d_{1}\right)+J_{k-n}\left(\beta_{1} d_{1}\right)\right]\left[T_{k}\left(\beta_{1} a_{1}\right) P_{k m}-H_{k}^{(1)}\left(\beta_{1} a_{1}\right) \lambda_{k m}\right] \\
\Delta_{m n}^{\prime}=\sum_{k=0}^{\infty}\left[R_{k}\left(\beta_{1} a_{1}\right) P_{k m}-J_{k}\left(\beta_{1} a_{1}\right) \lambda_{k m}\right] S_{k n} \\
\Lambda_{m n}=\frac{\delta_{n}^{(2)}}{2} \sum_{k=0}^{\infty} \varepsilon_{k}\left[J_{k-n}\left(\beta_{1} d_{1}\right)-(-1)^{n} J_{k+n}\left(\beta_{1} d_{1}\right)\right]\left[T_{k}\left(\beta_{1} a_{1}\right) Q_{k m}-H_{k}^{(1)}\left(\beta_{1} a_{1}\right) \mu_{k m}\right] \\
\Lambda_{m n}^{\prime}=\sum_{k=0}^{\infty}\left[R_{k}\left(\beta_{1} a_{1}\right) Q_{k m}-J_{k}\left(\beta_{1} a_{1}\right) \mu_{k m}\right] T_{k n} \\
\Delta_{m}^{\prime \prime}=\frac{\varepsilon_{m}}{2} \sum_{n=0}^{\infty}(-1)^{n} \delta_{n}^{(1)}\left[H_{m+n}^{(1)}\left(\beta_{1} d_{3}\right)+(-1)^{n} H_{m-n}^{(1)}\left(\beta_{1} d_{3}\right)\right]\left[J_{m}\left(\beta_{2} a_{2}\right) R_{m}\left(\beta_{1} a_{2}\right)-\gamma R_{m}\left(\beta_{2} a_{2}\right) J_{m}\left(\beta_{1} a_{2}\right)\right] \\
\Delta_{m}^{\prime \prime \prime}=\frac{\varepsilon_{m}}{2} \sum_{n=0}^{\infty}\left[J_{m}\left(\beta_{2} a_{2}\right) R_{m}\left(\beta_{1} a_{2}\right)-\gamma R_{m}\left(\beta_{2} a_{2}\right) J_{m}\left(\beta_{1} a_{2}\right)\right]\left[H_{m+n}^{(1)}\left(2 \beta_{1} d_{3}\right)+(-1)^{n} H_{m-n}^{(1)}\left(2 \beta_{1} d_{3}\right)\right] \\
\Lambda_{m}^{\prime \prime}=\frac{\varepsilon_{m}}{2} \sum_{n=0}^{\infty}(-1)^{n} \delta_{n}^{(2)}\left[H_{m+n}^{(1)}\left(\beta_{1} d_{3}\right)-(-1)^{n} H_{m-n}^{(1)}\left(\beta_{1} d_{3}\right)\right]\left[J_{m}\left(\beta_{2} a_{2}\right) R_{m}\left(\beta_{1} a\right)-\gamma R_{m}\left(\beta_{2} a\right) J_{m}\left(\beta_{1} a\right)\right]
\end{gathered}
$$




$$
\begin{array}{ccc}
\Lambda_{m}^{\prime \prime \prime}= & \frac{\varepsilon_{m}}{2} \sum_{n=0}^{\infty}\left[-J_{m}\left(\beta_{2} c\right) R_{m}\left(\beta_{1} c\right)+\gamma R_{m}\left(\beta_{2} c\right) J_{m}\left(\beta_{1} c\right)\right]\left[H_{m+n}^{(1)}\left(2 \beta_{1} d_{3}\right)+(-1)^{n} H_{m-n}^{(1)}\left(2 \beta_{1} d_{3}\right)\right] \\
S_{k n}=\frac{\varepsilon_{k}}{2}\left\{(-1)^{k}\left[(-1)^{n} H_{k-n}^{(1)}\left(\beta_{1} d_{2}\right)-H_{k+n}^{(1)}\left(\beta_{1} d_{2}\right)\right]+\left[(-1)^{n} H_{k-n}^{(1)}\left(\beta_{1} d_{4}\right)-H_{k+n}^{(1)}\left(\beta_{1} d_{4}\right)\right]\right\} \\
T_{k n}=\frac{\varepsilon_{k}}{2}\left\{(-1)^{k}\left[(-1)^{n} H_{k-n}^{(1)}\left(\beta_{1} d_{2}\right)-H_{k+n}^{(1)}\left(\beta_{1} d_{2}\right)\right]+\left[H_{k+n}^{(1)}\left(\beta_{1} d_{4}\right)-(-1)^{n} H_{k-n}^{(1)}\left(\beta_{1} d_{4}\right)\right]\right\} \\
\Phi_{m}=J_{m}\left(\beta_{2} a_{2}\right) T\left(\beta_{1} a_{2}\right)-\gamma R_{m}\left(\beta_{2} a_{2}\right) H_{m}^{(1)}\left(\beta_{1} a_{2}\right) & U_{n}=\varepsilon_{n} \mathrm{i}^{n}\left[\xi_{1}^{-1} \cos n\left(\frac{\pi}{2}-\alpha\right)+\xi_{1} \cos n\left(\frac{\pi}{2}+\alpha\right)\right] \\
P_{k m}=\sum_{l=0}^{\infty} \frac{J_{l}\left(\beta_{1} a_{1}\right)}{R_{l}\left(\beta_{1} a_{1}\right)} \lambda_{k l} \lambda_{l m} & \Phi_{m}^{\prime}=-J_{m}\left(\beta_{2} a_{2}\right) R_{m}\left(\beta_{1} a_{2}\right)+\gamma R_{m}\left(\beta_{2} a_{2}\right) J_{m}\left(\beta_{1} a_{2}\right) \\
Q_{k m}=\sum_{l=0}^{\infty} \frac{J_{l}\left(\beta_{1} a_{1}\right)}{R_{l}\left(\beta_{1} a_{1}\right)} \mu_{k l} \mu_{l m} & W_{m}=\varepsilon_{m} \mathrm{i}^{m}\left[\xi_{2}^{-1} \cos m\left(\frac{\pi}{2}-\alpha\right)+\xi_{2} \cos m\left(\frac{\pi}{2}+\alpha\right)\right] \\
V_{n}=\varepsilon_{n}{ }^{n}\left[\xi_{1}^{-1} \sin n\left(\frac{\pi}{2}-\alpha\right)+\xi_{1} \sin n\left(\frac{\pi}{2}+\alpha\right)\right] & X_{m}=\varepsilon_{m} \mathrm{i}^{m}\left[\xi_{2}^{-1} \sin m\left(\frac{\pi}{2}-\alpha\right)+\xi_{2} \sin m\left(\frac{\pi}{2}+\alpha\right)\right]
\end{array}
$$

The constants $A_{n}, B_{n}, C_{n}, D_{n}$ can be obtained with truncation order n, and $E_{n}, F_{n}, G_{n}, H_{n}$ can be obtained by inserting $A_{n}, B_{n}, C_{n}, D_{n}$ into equations (37) (39) (41) (43). The errors between the numerical results and the theoretical results can be checked with the stress-free condition on boundary $L$ and the continuity condition on $\bar{L}$ and $C$.

When $\varphi=0$, we will obtain the solution without the hill. In the situation, $\Delta_{m n}^{\prime}=0, \Lambda_{m n}^{\prime}=0$, and the right values of equation (45) and equation (47) are equal to zero, which lead to $A_{n}=0, B_{n}=0$. Finally the solution simplify to the known solutions [4] as following:

$$
\begin{array}{r}
\Phi_{m} C_{m}+\sum_{n=0}^{\infty} \Delta_{m n}^{\prime \prime \prime} C_{n}=\left[\gamma R_{m}\left(\beta_{2} a_{2}\right) J_{m}\left(\beta_{1} a_{2}\right)-J_{m}\left(\beta_{2} a_{2}\right) R_{m}\left(\beta_{1} a_{2}\right)\right] W_{m} \\
m=0,1,2,3 \ldots \\
\Phi_{m}^{\prime} D_{m}+\sum_{n=0}^{\infty} \Lambda_{m n}^{\prime \prime \prime} D_{n}=\left[J_{m}\left(\beta_{2} a_{2}\right) R_{m}\left(\beta_{1} a_{2}\right)-\gamma J_{m}\left(\beta_{1} a_{2}\right) R_{m}\left(\beta_{2} a_{2}\right)\right] X_{m} \\
m=1,2,3 \ldots
\end{array}
$$

When $\gamma=1$ and $\beta_{1}=\beta_{2}$, we will obtain the solution without the underground inclusion. $\Delta^{\prime \prime}{ }_{m n}=0$, $\Delta^{\prime \prime \prime}{ }_{m n}=0, \Lambda^{\prime \prime}{ }_{m n}=0, \Lambda^{\prime \prime \prime}{ }_{m n}=0$, and the right values of equation (46) and equation (48) are equal to zero, which lead to $C_{n}=0, D_{n}=0$. Finally, the solution simplify to the known solutions [5] as following:

$$
\begin{gathered}
\sum_{n=0}^{\infty} \Delta_{m n} A_{n}=\sum_{n=0}^{\infty} U_{n}\left[J_{n}\left(\beta_{1} a_{1}\right) \lambda_{n m}-R_{n}\left(\beta_{1} a_{1}\right) P_{n m}\right] \\
m=0,1,2,3 \ldots \\
\sum_{n=0}^{\infty} \Lambda_{m n} B_{n}=\sum_{n=0}^{\infty} V_{n}\left[J_{n}\left(\beta_{1} a_{1}\right) \mu_{n m}-R_{n}\left(\beta_{1} a_{1}\right) Q_{n m}\right] \\
m=1,2,3 \ldots
\end{gathered}
$$

\section{Numerical results}

Define a dimensionless parameter ud, representing displacement amplitude of stress-free surface.

$$
u_{d}=|u| / u_{0},(r, \theta) \in \Gamma, L
$$

Define dimensionless parameter $\eta$, representing frequency of incident wave.

$$
\eta=\frac{2 b}{\lambda}=\frac{\beta_{1} b}{\pi}
$$

where $\lambda$ represents incident wavelength. We study the effect of an underground circular inclusion under a circular-arc hill on ground motion with the dimensionless parameter $u_{d}$. 


\section{The effect of a hill and an inclusion on surface displacement.}

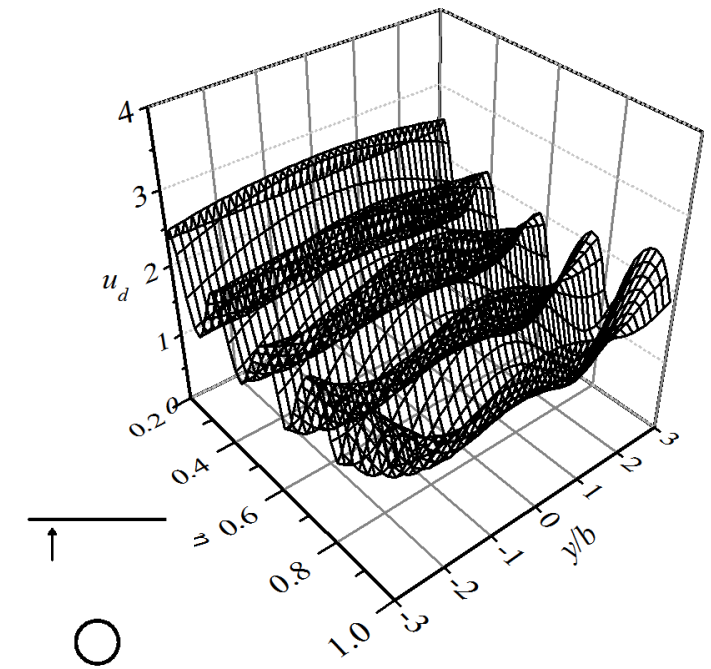

(a1) $h / b=0$

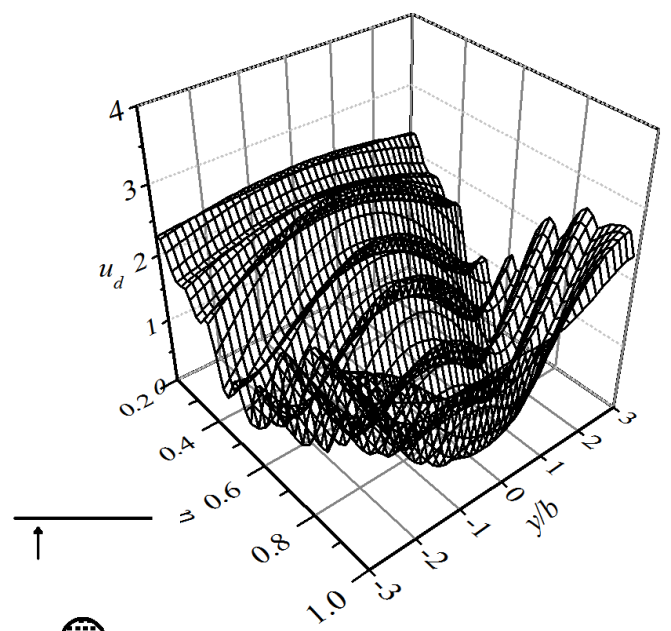

䚀

(b1 ) $h / b=0$

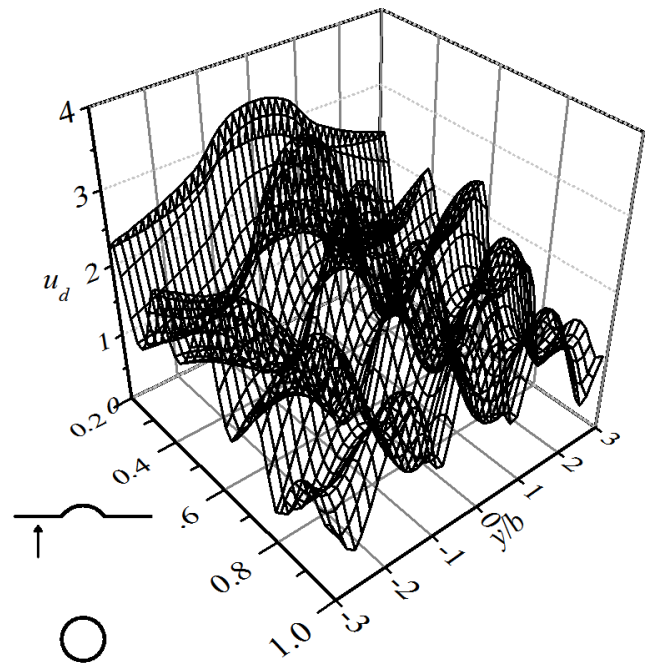

(a2) $h / b=0.5$

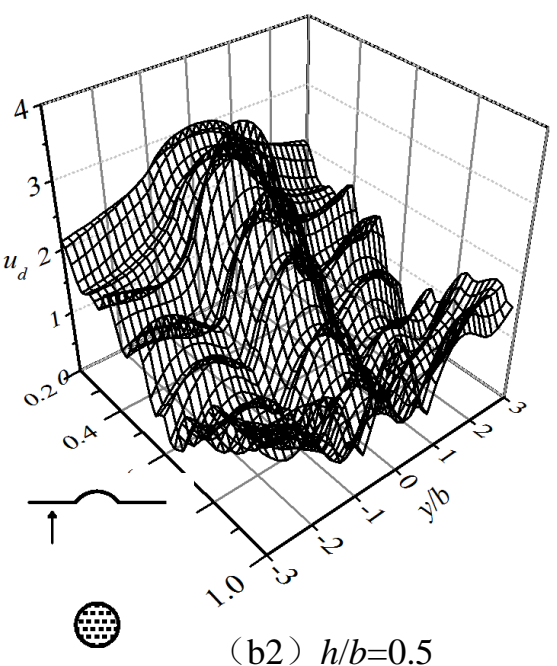

(b2) $h / b=0.5$

Fig. 4 The surface displacement amplitudes $u_{d}$ versus the dimensionless frequency $\eta$ and the dimensionless distance $y / b$ under vertical incidence for the following cases: (a1) and (b1) are the model with only an underground cavity and inclusion respectively, and (a2) and (b2) are the model with a hill $(h / b=0.5)$ above a cavity and an inclusion respectively. The buried depth and radius of the subsurface structures above $d_{3}=6 b, a_{2}=b$.

In this section, the surface displacement amplitudes of a hill above an underground structure under vertical incident SH wave are obtained. Furthermore, to discuss the respective effect of the hill and the underground structures, three degraded models are discussed as well: one with only the cavity, one with only the inclusion, and last one with only the hill.

Fig4a1 shows that there are periodic peaks and troughs due to large and small displacement amplitudes on the surface exhibited. To interpreter the distribution law, we analyze $u_{d}$ at the point $y=0$ firstly. We know that if the phase difference of the incident wave reaching surface directly and its reflections from the boundary of the cavity is $2 n \pi$ and $(2 n-1) \pi$ ( $n$ represents a positive integer), their superposition on the ground would be maximum and minimum respectively. The observation that these phase difference corresponding to a maximum or minimum displacement is a group of arithmetic progression suggests that they relate to the periodicity of the peaks and troughs. To support the conjecture, from phase difference $\omega \Delta t=2 n \pi$ and $(2 n-1) \pi$ we obtain the equation:

$$
\eta=n a_{2} /\left(d_{3}-a_{2}\right)
$$




$$
\eta=(2 n-1) a_{2} / 2\left(d_{3}-a_{2}\right)
$$

where equation (49) is same with that concluded in the previous work [4]. Substituting the known parameters into the equation (49) and (50), the frequencies estimated (peaks: 0.2, 0.4, 0.6, 0.8, 1.0 and troughs: $0.3,0.5,0.7,0.9)$ are in good agreement with that shown in fig $4 \mathrm{a} 1$. Next, the propagation path length of the reflections reaching ground surface $-3 \leq y / b \leq 3$ between the boundary of the cavity and ground surface is unequal, symmetrical by the line $y=0$. This leads to the bent ripples in fig $4 \mathrm{a} 1$.

Compared with the cavity, fig $4 \mathrm{~b} 1$ shows the ground displacement with a inclusion filled with softer medium with respect to the surrounding medium $\left(\gamma=\mu_{2} / \mu_{1}=1 / 4, \quad c_{2} / c_{1}=1 / 2\right)$. The displacement amplitudes in the surface direction $y$ change. There are also several peaks in the figure. Unlike the peaks in the fig $4 \mathrm{a} 1$, the distances between them is not equal. However, specially, the distribution of the peaks at low frequencies of $\lambda \geq 5 a_{2}$ can be estimated with reasonable accuracy by equation (49).

Fig 5 shows that the maximum displacement amplitude appear on the hilltop at the frequencies we

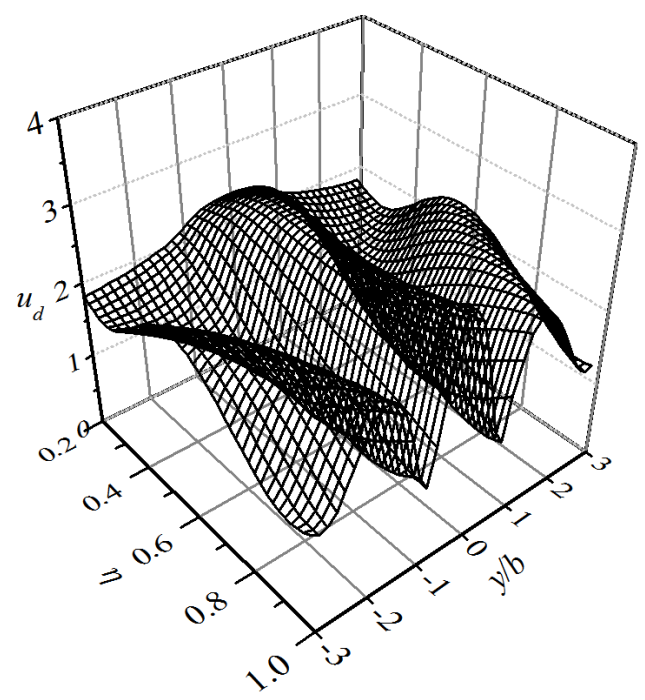

Fig. 5 Surface displacement amplitudes with a hill $(h / b=0.5)$ discussed, and the vibration near the hill rims $(y / b= \pm 1)$ is weakened with the frequencies increasing.

With combining the characters of the effect of the underground structure and the hill we analyze their combined effect on ground motion. Fig4a 2 and fig $4 \mathrm{~b} 2$ show that the surface displacement would be large (small) in the region where it is amplified (weakened) both in its two degraded models (as the point $\eta=0.4, y=0$ shown in fig $4 \mathrm{a} 2$, corresponding to that shown in fig 4a1 and fig 5). So we can easily estimate the dangerous frequencies at the hilltop under vertical incidence with equation (49). Here we conclude that the effect can be seen as the superposition of the effect of the inclusion and the hill each other qualitatively. Yet the superposition effect is

not equal to the sum or the product of that of each other.

\section{The effect of the medium of inclusion and the shape of hill on surface displacement.}

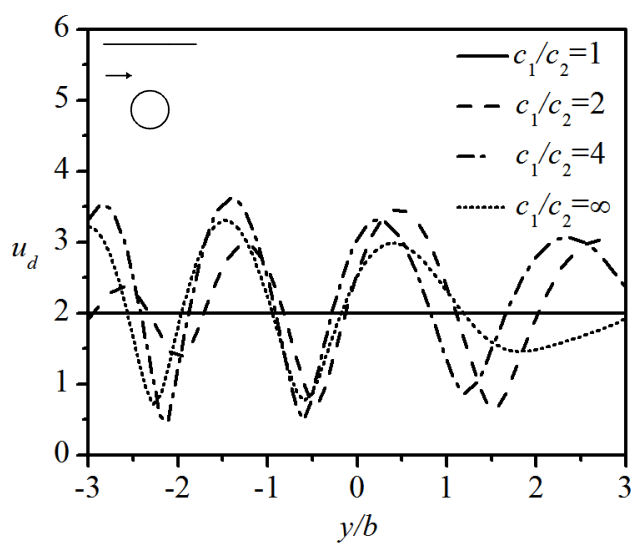

(a1) $\alpha=0^{\circ}, h / b=0$

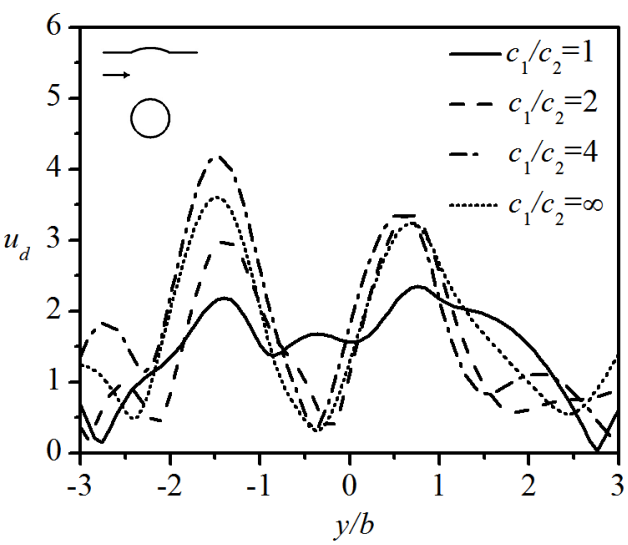

(a2) $\alpha=0^{\circ}, h / b=0.25$ 


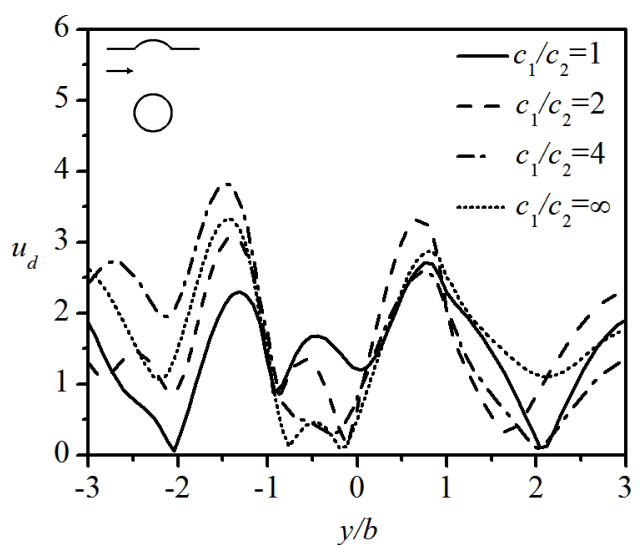

(a3) $\alpha=0^{\circ}, h / b=0.5$

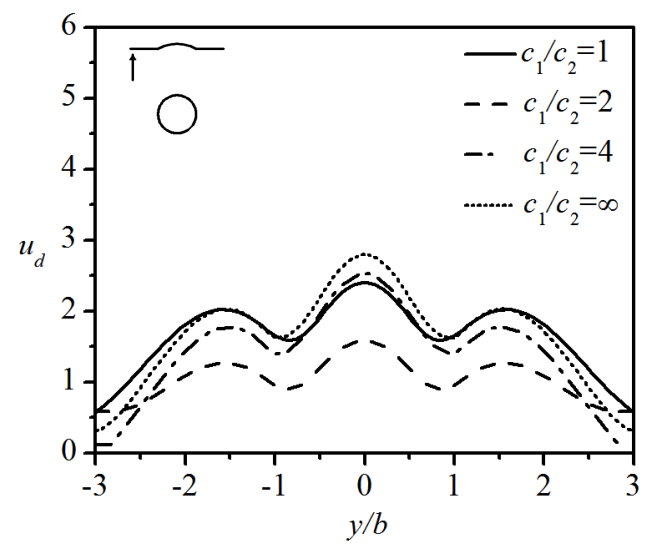

(b2) $\alpha=90^{\circ}, h / b=0.25$

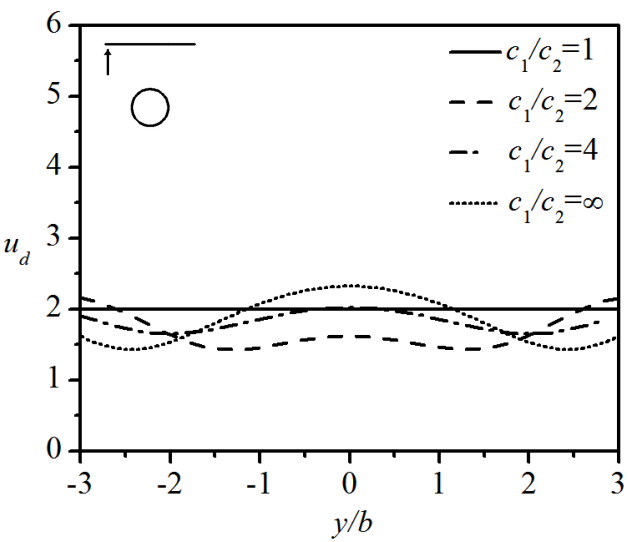

(b1) $\alpha=90^{\circ}, h / b=0$

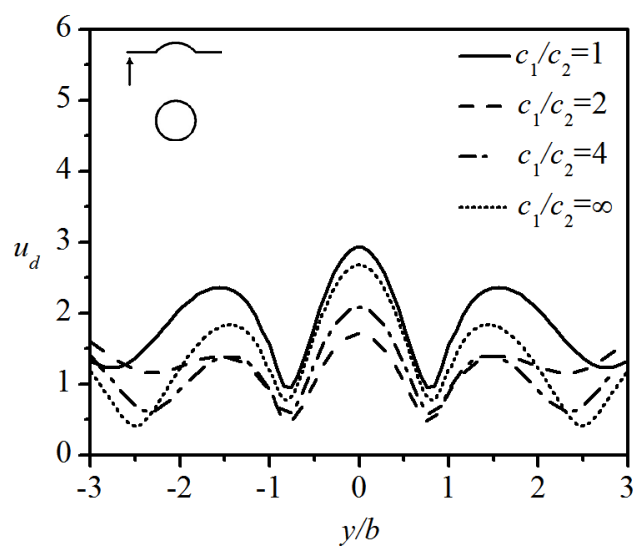

(b3) $\alpha=90^{\circ}, h / b=0.5$

Fig. 6 Several surface displacement amplitude obtained for the computed parameters as following: $\eta=1, h / b=0,0.25,0.5, d_{3}=6 b, a_{2}=b$, and the density and the wave velocity of the inclusion $\rho_{1} / \rho_{2}=1, c_{1} / c_{2}=1,2,4, \infty$, where $h / b=0$ represents the results without the hill, and $c_{1} / c_{2}=1$ presents the model without a inclusion, $c_{1} / c_{2}=2,4$ presents a soft and softer inclusions respectively, and $c_{1} / c_{2}=\infty$ presents a cavity. Fig $6 a 1 \sim a 3$ is for horizontal incidence, and fig $6 b 1 \sim b 3$ is for vertical incidence.

Fig $6 a 1$ is in the circumstances without the hill. It can be noted that the displacement with the soft inclusion and the tunnel is large around two points $y / b=-1.4,0.5$; when the inclusion is soft enough (like $c_{1} / c_{2}=4$ ), its effect is equivalent in function to a cavity on $y / b \in(-3,0)$; Simply, the tough inclusion cause a weak surface displacement. Fig $6 \mathrm{a} 2$ and fig $6 \mathrm{a} 3$ indicate that, special for the soft inclusion and the tunnel, the surface regions vibrated greatly with a hill is the same with that without a hill nearly; the tough inclusion only causes a large displacement amplitude at surface $y / b=0.5$ when $h / b=0.5$.

Next we analyze the situation of vertical incidence. Fig6b1 b3 show that the softer the inclusion, the larger surface displacement amplitude is. For example, in fig 6b3, $u_{d} 1.6$ at $c_{1} / c_{2}=2$ while 2.1 and 2.6 at $c_{1} / c_{2}=4$ and $\infty$, increasing $31.1 \%$ and $62.5 \%$ respectively. At hill rims $(y / b= \pm 1)$, the greater height-to-width, the weaker the $u_{d}$ is. As hill rims in fig 6b3, the surface displacement amplitude is unchanged nearly with changing the softness and hardness of the inclusion. The hard inclusion weakens the ground vibration above it, less than 0.5 , decreasing $75 \%$ than free surface of a half-space.

\section{Conclusion}

The analytical solution of scattering of $\mathrm{SH}$ wave by an underground inclusion under an arc-circular hill has been obtained in this paper. It can be concluded that:

1) The effect of a hill and an inclusion on surface displacement amplitude can be seen as the superposition effect of each other qualitatively. Generally speaking, the displacement would be large 
in the common amplified region of the two degenerated models: the one without the hill, the other one without the inclusion.

2) The computation results show that flat ground surface above a tunnel vibrate greatly and weakly under vertical incidence at periodic frequencies, and the amplified dimensionless frequencies are obtained $\eta=n b /\left(d_{3}-a_{2}\right)$. Specially, it is also applied in the circumstance of a soft inclusion at low frequency band $\left(\eta \leq 2 b / 5 a_{2}\right)$. Dangerous region can be forecasted by combining with concentration of dangerous region on the hill.

3) The ground motion is remarkably affected by softness and hardness of inclusion. Under horizontal incidence, the soft inclusion (like $c_{1} / c_{2} \geq 2$ ) makes it violent on the same position with the circumstance of the tunnel. It behaves as a simple kinetic characteristic under vertical incidence.

\section{Acknowledgements}

This work was financially supported by the Scientific Research Fund of Institute of Engineering Mechanics, China Earthquake Administration (Grant No.2014B06).

\section{References}

[1] J.W. Liang, H. Luo, and V. W. Lee, in: Scattering of plane SH waves by a circular-arc hill with a circular tunnel, Earthquake Science, Beijing, China (2004), in press.

[2] D.K. Liu, G.Q. Wang, in: Antiplane SH-deformation of a semi-cylindrical hill above a subsurface cavity, Acta Mechanica Sinica, Beijing, China (2006), in press.

[3] I.A. Stegun and M. Abramowitz: Handbook of mathematical functions with formulas, graphs, and mathematical tables (US GPO, USA 1972).

[4] C. Smerzini, J. Avilés, R. Paolucci, et al, in: Effect of underground cavities on surface earthquake ground motion under SH wave propagation, Earthquake Engineering \& Structural Dynamics, W Sussex, England (2009), in press.

[5] X.M. Yuan and Z.P. Liao, in: Surface motion of a cylindrical hill of circular-arc cross-section for incident plane SH waves, Soil Dynamics \& Earthquake Engineering, Oxon, England (1996), in press. 